\title{
La mise en ouvre des nouvelles orientations de la politique de prévention des inondations en France, adoptées en janvier 1994
}

\author{
Noël Godard \\ ICPC, Sous-directeur de la gestion des eaux au ministère de l'environnement
}

\begin{abstract}
L'importance des inondations qu'a connues la France de septembre 1992 à janvier 1994 et la gravité de leurs conséquences en terme de vies humaines (près d'une centaine de victimes sur moins de dix huit mois) et de dommages matériels (évalués à plus de dix milliards de francs) ont conduit les Pouvoirs Publics à redéfinir en janvier 1994 les grands axes de la politique de prévention des inondations en France.

Les décisions correspondantes ont fait l'objet d'une réunion interministérielle présidée par le Premier Ministre le 24 janvier 1994. Le programme qui a été adopté lors de cette réunion a été évoqué au cours de la séance de clôture des $23^{\mathrm{c}}$ journées de l'hydraulique qui se sont tenues à Nîmes du 14 au 16 septembre 1994.
\end{abstract}

Les lecteurs de « La Houille Blanche » seront certainement intéressés de savoir où en est la mise en œuvre de ce programme près de neuf mois après son adoption.

Ce programme, prévu sur une durée de dix ans, comportait des mesures dans les quatre domaines suivants :

- la maîtrise de l'occupation des sols dans les zones inondables,

- la surveillance des inondations et la mise en sécurité des populations,

- un plan décennal de restauration et d'entretien des rivières,

- la réparation des dommages.

\section{La maîtrise de l'occupation des sols dans les zones inondables}

L'importance des dommages provoqués par les inondations de septembre 1992 à janvier 1994, résulte en grande partie d'une méconnaissance des risques d'inondation dans les décisions d'occupation des sols qui a conduit à construire dans des zones dangereusement exposées.

Elle a également été aggravée par la réduction de la capacité des champs naturels d'expansion des crues dont l'extension a été réduite au profit des villes et d'une agriculture intensive.

La mise en œuvre des procédures prévues pour réglementer l'occupation des sols dans les zones dangereuses (délimitation à l'initiative des préfets de «périmètres de risques" en application du code de l'urbanisme, élaboration par l'Etat de plans d'exposition au risque en application de la loi du 13 juillet 1982) concerne en 1994 moins de 700 communes, alors que plusieurs milliers de communes ont une partie de leur territoire en zone inondable.

Les «plans de surfaces submersibles » institués en 1935 et qui devaient permettre de préserver les capacités d'écoulement des crues et des champs d'inondation n'ont pas été élaborés dans toutes les vallées où ils étaient prévus (notamment dans le bassin de la Seine) et, quand ils ont été institués, n'ont pas toujours été bien respectés.

Les difficultés constatées proviennent en partie de réelles difficultés de procédures qui compliquent la mise en œuvre de ces outils juridiques et également de l'insuffisance des moyens mis à la disposition des services pour élaborer les cartes nécessaires.

Le programme adopté le 24 janvier a donc prévu un certain nombre de dispositions pour relancer très fortement l'action de l'Etat dans ce domaine:

- des instructions ont été données aux préfets par une circulaire interministérielle du 24 janvier 1994 pour clarifier les objectifs qui doivent être ceux de l'Etat dans la mise en cuvre des outils réglementaires existants en matière de gestion des zones inondables:

- interdire les constructions nouvelles dans les zones les plus exposées,

- préserver les zones inondables de tout occupation ou aménagement des sols susceptibles de réduire les capacités d'expansion des crues,

- interdire les nouveaux endiguements susceptibles de restreindre l'expansion des crues à l'exception de ceux qui sont nécessaires à la protection des quartiers urbains denses exposés aux crues.

L'Etat doit en effet exercer ses responsabilités dans ce domaine essentiel à la sécurité des populations.

- Des crédits nouveaux ont été mis en place pour financer la cartographie des zones inondables, la dotation annuelle qui était initialement de 15 millions de francs pour 1994 a déjà été portée à 22 millions de francs et le projet de budget pour 1995 prévoit de la porter à 40 millions de francs ; l'objectif est d'achever en 1997 la cartographie des zones inondables dans les 2000 communes les plus vulnérables; l'identification des 380 communes les plus exposées aux risques de crues torrentielles dans 24 départements du sud-est a été effectuée.

- Le dispositif juridique existant sera modernisé et simplifié ; le projet de loi relatif au renforcement de la protection de l'environnement, qui doit être discuté au Par- 
lement à l'automne 1994, prévoit de remplacer l'ensemble des plans existants par un seul document: le "plan de prévention des risques "; sa procédure d'instruction sera simple et déconcentrée; son contenu, modulable, pourra être adapté au contexte local et à l'état des connaissances. L'élaboration du guide méthodologique destiné aux services de l'Etat qui le mettront en œuvre au plan local a déjà été engagée de façon à pouvoir appliquer cette procédure unifiée dès que la loi sera adoptée.

\section{La surveillance des inondations et la mise en sécurité des populations}

Les maires sont responsables de la sécurité des populations durant les inondations. L'Etat, sans en avoir l'obligation légale, a mis en place sur $16000 \mathrm{~km}$ des principaux cours d'eau le long desquels est installée la majeure partie des populations exposées, des services d'annonce des crues afin d'informer les maires.

Ces services doivent être dotés d'outils modernes qui leur permettront de fournir des informations plus précises et plus précoces: stations de mesures télétransmises, modèles de prévision, mesure de la pluviométrie par radar dans les zones soumises à des précipitations intenses.

La dotation annuelle d'investissement du Ministère de l'Environnement qui y est consacrée a été portée de $18 \mathrm{mil}-$ lions de francs en 1993 à 25 millions de francs à partir de 1994. Cette dotation est abondée par les concours des collectivités locales concernées.

Le principe d'un programme de 5 nouveaux radars dans le sud-est de la France a été adopté. Une convention-cadre a été signée à cet effet entre Météo-France et le Ministère de l'Environnement. Le financement du premier de ces cinq radars qui sera implanté en Haute-Loire a été mis en place par le Ministère de l'Environnement. Le second radar devra compléter la couverture des départements de la vallée du Rhône, notamment le Vaucluse. Les trois suivants sont destinés à l'Aude et aux Pyrénées Orientales, à la Côte d'Azur et à la Corse.

Le Ministère de l'Intérieur modernisera parallèlement les moyens de transmission aux maires de l'alerte et des informations.

Une action spécifique a été engagée vis-à-vis des campings exposés aux risques: ces campings devront désormais mettre en cuvre des mesures d'information permanente, d'alerte et d'évacuation, conformément à un décret du 13 juillet 1994 qui a rendu obligatoire ces mesures.

\section{Un plan décennal de restauration et d'entre- tien des rivières}

Le mauvais entretien des rivières constitue un facteur aggravant des conséquences des inondations. Depuis plusieurs décennies, l'entretien n'est plus assuré sur de nombreuses rivières par les riverains qui en ont la charge dans le cas général. En ce qui concerne les rivières domaniales (environ $12000 \mathrm{~km}$ en France métropolitaine), l'Etat n'a pas toujours non plus mis en œuvre les moyens suffisants pour assurer complètement leur entretien.
C'est pourquoi le programme adopté le 24 janvier 1994 comporte un programme très important de rattrapage prévu sur 10 ans, d'un coût total évalué à environ 10,8 milliards de francs.

Ce programme doit conjuguer les efforts des propriétaires riverains, des collectivités locales et de l'Etat.

\subsection{Une amélioration de l'organisation}

Les propriétaires riverains seront incités à assumer leurs responsabilités, notamment lorsqu'ils seront regroupés en association syndicale. Le projet de loi relatif au renforcement de la protection de l'environnement a prévu d'instituer un outil technique de planification soumis à agrément préfectoral, « le plan simple de gestion ", qui permettra aux associations qui s'en doteront d'être prioritaire pour l'attribution d'aides publiques.

Les collectivités locales disposent par ailleurs grâce aux dispositions de l'article 31 de la loi sur l'eau du 3 janvier 1992, de possibilités juridiques accrues d'intervention dans l'entretien des rivières, les riverains n'ayant pas dans de nombreux cas les moyens suffisants pour assumer seuls leur responsabilité.

Une circulaire interministérielle du 17 août 1994 a rappelé l'ensemble des dispositions réglementaires et financières actuellement en vigueur relatives à l'intervention des propriétaires riverains et des collectivités locales en matière d'entretien des rivières et ouvrages hydrauliques de protection contre les inondations.

Trop souvent, en effet, celles-ci avaient été perdues de vue.

\subsection{De nouveaux moyens financiers}

Une part importante du financement du programme sera prise en charge par l'Etat.

Ce dernier engagera un effort de rattrapage sur les cours d'eau domaniaux : Voies Navigables de France mettra en particulier en œuvre un programme de 2 milliards de francs sur le domaine dont il a la gestion; une première tranche de 240 millions de francs sera engagée dès 1994.

Le Ministère de l'Environnement apportera des subventions (à un taux compris entre 20 et $25 \%$ ) pour aider au financement des investissements des maîtres d'ouvrage locaux destinés à rattraper le retard d'entretien des cours d'eau et des ouvrages de protection, restaurer les conditions de fonctionnement des champs d'expansion des crues et assurer la protection des zones urbanisées (une circulaire du 3 mai 1994 a défini les modalités d'intervention de l'Etat).

Le projet de budget pour 1995 prévoit que les dotations annuelles budgétaires du Ministère de l'Environnement consacrées à ces actions seront augmentées de 92 millions de francs par rapport aux dotations inscrites initialement dans le budget pour 1994 (soit une augmentation de $67 \%$ ).

Dès 1994 des dotations supplémentaires d'un montant de 74 millions de francs ont été mises en place.

Ces dotations supplémentaires ont notamment permis :

- d'engager un programme de restauration du lit de la Loire,

- de renforcer les dotations destinées aux départements d'outre-mer (Antilles et Réunion), 


\section{POLITIQUE DE PRÉVENTION DES INONDATIONS}

- de répondre aux demandes de financement les plus urgentes dans les départements du quart sud-est de la France.

Les Agences de l'Eau ont été sollicitées pour augmenter dès 1994 le volume des concours financiers qu'elles apportent aux études préalables et aux travaux de restauration des rivières et notamment à ceux destinés à réhabiliter les zones d'expansion des crues.

Une adaptation de leurs règles d'intervention, au travers d'un élargissement des assiettes des redevances qu'elles sont habilitées à percevoir, est en cours de mise au point pour élargir leurs capacités d'action, de façon à aider les initiatives des collectivités locales.

\section{La réparation des dommages}

Le mécanisme d'indemnisation des dommages aux biens assurables consécutifs aux inondations institué dans le cadre de la loi du 13 juillet 1982 a fait preuve de son efficacité lors des dernières inondations. En contrepartie de la surtaxe de $9 \%$ qu'elles prélèvent sur les primes d'assurances, les sociétés d'assurances ont versé au titre des inondations intervenues de septembre 1992 à janvier 1994 près de 5,5 milliards de francs d’indemnisations.

En revanche, le financement des réparations des équipements publics non assurés des communes et départements reste difficile pour les collectivités locales les moins fortunées. Pour faire face aux dommages aux équipements publics, l'Etat a dégagé des financements exceptionnels. Au total l'Etat a supporté environ 1,5 milliard de francs au titre des inondations de septembre 1992 à janvier 1994 pour la réparation des équipements publics.

Des études et concertations ont été engagées pour mettre en place un système permanent de mutualisation des risques entre les collectivités publiques. 